ORIGINAL ARTICLE

\title{
Combining isometric knee extension exercises with hip adduction or abduction does not increase quadriceps EMG activity
}

\author{
J Hertel, J E Earl, K K W Tsang, S J Miller
}

Br J Sports Med 2004;38:210-213. doi: 10.1136/bjsm.2002.003277

See end of article for authors' affiliations .....................

Correspondence to: J Hertel, Penn State University, Department of Kinesiology, 269A Recreation Building University Park, PA 16802, USA; jnh3@psu.edu

Accepted 15 April 2003

\begin{abstract}
Objective: To determine if the combined isometric contractions of knee extension/hip adduction and knee extension/hip abduction will elicit a different quadriceps and gluteus medius electromyographic (EMG) pattern as compared to isometric contraction of a uniplanar knee extension exercise.

Methods: Eight healthy young adult volunteers without history of knee or quadriceps injury participated. Surface EMG data were collected from the vastus medialis oblique (VMO), vastus lateralis (VL), and gluteus medius (Gmed) muscles of the dominant leg of each subject during three single leg, weight bearing, isometric exercises (uniplanar knee extension, knee extension/hip adduction, knee extension/hip abduction). All exercises were performed at a position of $60^{\circ}$ knee flexion. Three trials lasting $5 \mathrm{~s}$ each were performed for each of the three exercises. EMG data from each muscle were integrated and the maximum root mean square activity over a $0.5 \mathrm{~s}$ window for each trial was averaged. Analyses of variance were performed with exercise (straight extension, extension/adduction, extension/abduction) as the independent variable and $\mathrm{VMO}, \mathrm{VL}$, and Gmed activity and VMO:VL ratio as dependent variables. Results: A significant main effect for exercise was found for the VMO $(p=0.006)$ and $\mathrm{VL}(p=0.02)$, but not the Gmed ( $p=0.25$ ) or the VMO:VL ratio $(p=0.13)$. For the $V M O$ and $V L$, the uniplanar knee extension task produced significantly more EMG activity than the extension/adduction or extension/abduction tasks. Conclusions: Uniplanar knee extension exercises may be more appropriate than combining isometric knee extension exercises with hip adduction or abduction when eliciting maximal VMO and VL contractions.
\end{abstract}

of medial rotation of the hip compared to neutral or laterally rotated positions. They did not, however, report measures of GMed activity.

We are unaware of previous investigations that have assessed muscle activation of the quadriceps and gluteus medius muscles with combined knee and hip motions during single leg, weight bearing exercises. Therefore, the purpose of this study was to determine if combined single leg isometric contractions of knee extension/hip adduction and knee extension/hip abduction altered VMO, VL, and GMed EMG activity compared to uniplanar knee extension isometric contractions.

\section{METHODS \\ Subjects}

Eight healthy young adult volunteers (five males, three females, age $=24 \pm 2.5$ years, mass $=67.2 \pm 10.3 \mathrm{~kg}$, height $=$ $169.5 \pm 4.7 \mathrm{~cm}$ ) without history of knee or quadriceps injury participated. All subjects read and signed an informed consent form approved by the Pennsylvania State University Institutional Review Board prior to participation in the study.

\section{Instrumentation}

Surface electromyography (EMG) was used to quantify muscle activation of the VMO, VL, and GMed muscles. EMG hardware and software was manufactured by Biopac (Santa Barbara, CA). The EMG signals were analysed using Acknowledge Software version 3.5 (Biopac Systems, Santa Barbara, CA). The data was high pass filtered at $75 \mathrm{~Hz}$. The following parameters were used: band width $10-500 \mathrm{~Hz}$,

Abbreviations: EMG, electromyographic; Gmed, gluteus medius; PFPS, patellofemoral pain syndrome; VL, vastus lateralis; VMO, vastus medialis oblique 
input impedance $2 \mathrm{M} \Omega$ (differential), common mode rejection ratio $110 \mathrm{~dB}$, maximum input voltage $\pm 10 \mathrm{~V}$, sampling rate $1000 \mathrm{~Hz}$, gain 1000 .

\section{Procedures}

Prior to electrode placement, the skin was prepared by shaving and vigorously cleaning the appropriate areas with alcohol wipes. The VMO electrodes were placed at an angle of approximately $55^{\circ}$ to the long axis of the femur at a location that was over the muscle belly when the knee was in $60^{\circ}$ of flexion. ${ }^{20}$ The VL electrodes were placed proximal to the distal tendon over the area of greatest muscle bulk. The Gmed electrodes were placed over the proximal third of the line between the iliac crest and the greater trochanter. The interelectrode distance for all three muscles was $2 \mathrm{~cm}$. A ground electrode was placed on the tibial crest of the test leg. The same experienced researcher (JEE) applied the electrodes in a consistent manner to all subjects.

Subjects performed three types of weight bearing, isometric exercises with their dominant limbs: uniplanar knee extension, knee extension/hip adduction, and knee extension/hip abduction. Three trials of $5 \mathrm{~s}$ each were performed for each of the exercises. Each trial was separated by 2 min of rest. Verbal encouragement to perform maximally was given to subjects for all trials. Supramaximal electrical stimulation was purposefully not used because we wanted to study maximal "voluntary" isometric contractions, not absolute maximal muscle capacity.

For the uniplanar knee extension exercises, a custom-made testing apparatus was used. This consisted of a $30^{\circ}$ angled platform covered with a non-slip surface. Participants stood with their back against the wall and their test leg on the platform in front of them with their knee positioned in $60^{\circ}$ of flexion (fig 1). This position was chosen because it has been previously shown that greatest activation of the VMO is achieved at $60^{\circ}$ of knee flexion during the weight bearing exercise. ${ }^{6}$ Knee joint angle was measured with a standard goniometer prior to the start of each trial to ensure consistent positioning across the trials of all subjects. The distance of the platform from the wall was adjustable to account for differences in subject height and leg length. A heavy rubber mat was placed in front of the platform to prevent it from sliding. Shoulder straps ensured that subjects did not move their trunk, hip, or knee when they performed the isometric contractions. Subjects were instructed to lift their contralateral foot and to maximally push up and back into the wall with their test leg.

The knee extension/hip adduction task used the same setup with the addition of a towel roll between subjects' knees (fig 2). Subjects were instructed to maximally contract their test leg and simultaneously squeeze both knees together (hip adduction). For the knee extension/hip abduction task subjects were positioned with their test leg on the angled platform and their non-test side against the wall. Subjects were instructed to drive their non-test hip into the wall (causing hip abduction contralaterally) with maximal effort as they performed maximal isometric knee extension on their test leg (fig 3).

\section{Data processing}

EMG data for each muscle were integrated and the maximum root mean square (RMS) activity over a $0.5 \mathrm{~s}$ window for each trial was calculated and used as the dependent variables. The data were not normalised because all comparisons made in this study were within-day comparisons of single muscles and the electrodes were left in place for all tests. ${ }^{27}$

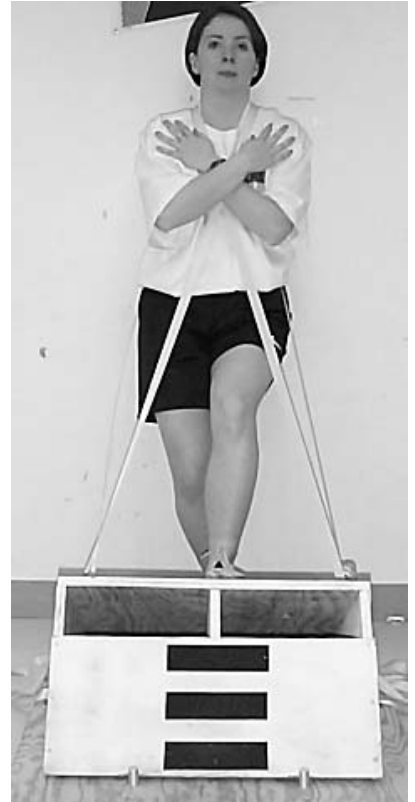

Figure 1 Straight knee extension exercise.

\section{Statistical analysis}

Four separate analyses of variance with repeated measures were performed with exercise (uniplanar knee extension, knee extension/hip adduction, knee extension/hip abduction) as the independent variable and normalised maximal RMS activity of the VMO, VL, and GMed, and the VMO:VL ratio as the dependent variables. Tukey's post hoc tests were used to identify specific significant differences in the presence of a significant ANOVA. The level of significance was preset at $\mathrm{p}<0.05$ for all analyses.

\section{RESULTS}

Muscle activation patterns were significantly different between the three exercises for the VMO $\left(\mathrm{F}_{2,14}=7.38\right.$, $\mathrm{p}=0.006)$ and the VL $\left(\mathrm{F}_{2,14}=5.17, \mathrm{p}=0.02\right)$. Post hoc

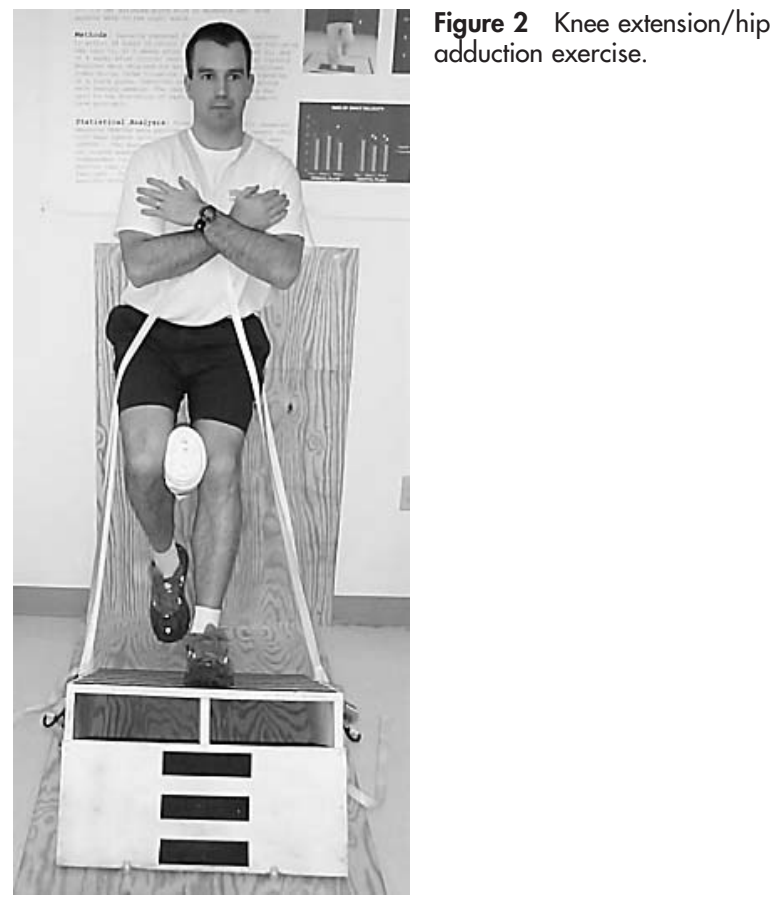




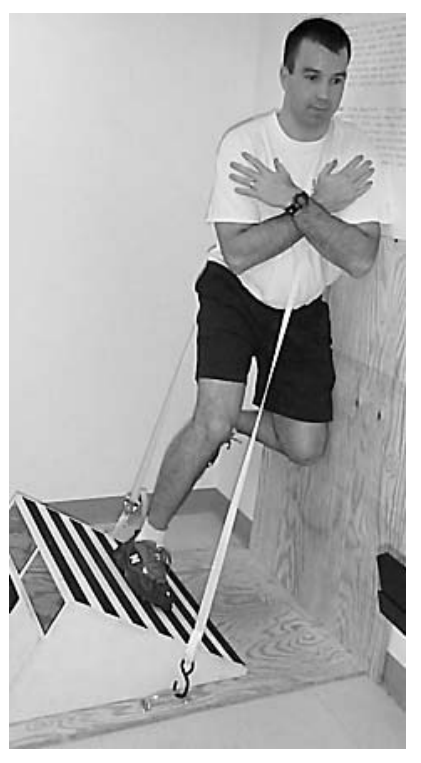

Figure 3 Knee extension/hip abduction exercise.

analysis revealed that for the VMO and VL both the knee extension/hip adduction task and the knee extension/hip abduction task produced significantly less EMG activity than the uniplanar knee extension task $(\mathrm{p}<0.03)$. There were no significant differences between the knee extension/hip adduction and knee extension/hip abduction exercises for either the VMO or VL. There were no significant differences for GMed activity $\left(\mathrm{F}_{2,14}=1.53, \mathrm{p}=0.25\right)$ or VMO:VL ratio between exercises $\left(\mathrm{F}_{2,14}=2.39, \mathrm{p}=0.13\right)$. Means and standard deviations for all dependent measures may be seen in table 1 .

\section{DISCUSSION}

Our primary finding was that maximal surface EMG activity of the VMO and VL was greater when performing weight bearing, isometric, uniplanar knee extension exercises compared to exercises combining knee extension with hip abduction or adduction. The VMO and VL responded similarly in our study as the VMO:VL ratio was not significantly different between the three exercises.

Our findings are inconsistent with previous literature that identified enhanced VMO activity with combined knee extension and hip adduction during weight bearing. ${ }^{419} 23$ We had subjects perform the knee extension/hip adduction in single leg stance whereas previous studies have utilised bilateral squatting exercises. ${ }^{4}{ }^{19} 23$ We chose single leg knee extension/hip adduction because our knee extension/hip abduction exercise required single leg stance. It could be that these novel, single leg stance exercises required

Table 1 Mean maximum iEMG activity $( \pm S D)$ of the three muscles during the different exercises

\begin{tabular}{llll}
\hline & $\begin{array}{l}\text { Straight knee } \\
\text { extension }\end{array}$ & $\begin{array}{l}\text { Knee extension/ } \\
\text { hip adduction }\end{array}$ & $\begin{array}{l}\text { Knee extension/ } \\
\text { hip abduction }\end{array}$ \\
\hline $\begin{array}{l}\text { Vastus medialis } \\
\text { oblique (mv) }\end{array}$ & $1.25^{*}(0.53)$ & $0.96(0.40)$ & $0.89(0.31)$ \\
$\begin{array}{l}\text { Vastus lateralis } \\
\text { (mv) }\end{array}$ & $0.42^{*}(0.15)$ & $0.37(0.13)$ & $0.35(0.13)$ \\
$\begin{array}{l}\text { Gluteus medius } \\
\text { (mv) }\end{array}$ & $0.15(0.10)$ & $0.13(0.007)$ & $0.16(0.008)$ \\
VMO:VL ratio & $3.00(1.04)$ & $2.58(0.58)$ & $2.58(0.43)$ \\
\hline
\end{tabular}

${ }^{*}$ Measures significantly greater $(p<0.05)$ with straight knee extension exercise compared to the other exercises. volunteers to focus on maintaining their balance and they thus were concentrating more on recruiting postural stabilisers than the quadriceps. Another explanation could be that concentration on producing horizontally directed force (hip abduction and adduction) may have diminished the ability to produce vertically directed force (knee extension). Previously, Yamashita $^{28}$ suggested that VMO activity is enhanced by combining knee extension with hip extension. This is most likely more easily accomplished in the closed kinetic chain with a pure sagittal plane exercise than with multiplanar exercises.

Previous studies demonstrating increased VMO activity with knee extension/hip adduction activities used isotonic exercises $^{41923}$ while we studied isometric exercises. Isotonic squatting exercises may lead to increased VMO activity because the VMO must be more active in the dynamic stabilisation of the patella during knee movement. While more dynamic activities are certainly a necessity in advanced rehabilitation, uniplanar knee extension isometric exercise may be more appropriate during early rehabilitation where retraining of the VMO is critical to restoring normal patellofemoral mechanics.

Patellofemoral dysfunction may also be related to inadequate control of femoral rotation. ${ }^{25} 26$ Excessive internal rotation of the femur may contribute to increased lateral tracking of the patella. During weight bearing activities, the GMed eccentrically controls femoral internal rotation. ${ }^{29}$ The GMed has also been shown to be an important contributor to pelvic stability during weight bearing. ${ }^{30}$ Inadequate strength or recruitment of the GMed alone, or in combination with VMO dysfunction, may lead to PFPS. While we did not find significant differences in GMed activity during our three exercises, the knee extension/hip abduction task was associated with the greatest amount of GMed activity. Because this task was isometric and did not involve dynamic hip and knee flexion (and associated femoral internal rotation), it may not have caused maximal GMed activity.

Our study was not without limitations. We only examined variables of EMG amplitude and did not measure timing variables. Onset of muscle activation has been shown to be altered in PFPS patients ${ }^{70-13}$ and clinicians should be mindful of these changes when implementing neuromuscular rehabilitation programs. Another limitation is that our comparisons were of non-normalised EMG signals. While using non-normalised signals allowed us to answer our research question, it may limit the ability to compare our results to those of others who analysed normalised EMG signals. Lastly, our sample size was not large $(n=8)$ and the generalisability of our findings to more diverse populations may be limited. These data provide preliminary answers to our research questions, however a larger study utilising more subjects both with and without PFPS would better control for the considerable intersubject variability of surface EMG, provide for increased statistical power, and expand the generalisability of the results.

\section{CONCLUSION}

We found that there was significantly greater surface EMG activity of the VMO and VL during weight bearing, isometric

\section{Take home message}

If eliciting maximal activity of the $\mathrm{VMO}$ and $\mathrm{VL}$ during weight bearing isometric exercise is a clinician's rehabilitation goal, a uniplanar knee extension exercise appears to be more appropriate than combining hip abduction or adduction with knee extension. 
uniplanar knee extension exercises than with either combined knee extension/hip adduction or knee extension/hip abduction exercises. Further research investigating the muscle activation patterns during various isometric and isotonic exercises commonly prescribed for PFPS is warranted.

\section{Authors' affiliations}

J Hertel, S J Miller, Penn State University, University Park, PA, USA Jennifer E Earl, University of Wisconsin-Milwaukee, Milwaukee, WI, USA

K K W Tsang, Michigan State University, East Lansing, MI, USA

Conflict of interest: none declared.

\section{REFERENCES}

1 Moller BN, Krebs B, Tidemand-Dal C, et al. Isometric contractions in the patellofemoral pain syndrome. An electromyographic study. Arch Orthop Trauma Surg 1986;105:24-7.

2 Thomee R, Renstrom P, Karlsson J, et al. Patellofemoral pain syndrome in young women. II. Muscle function in patients and healthy controls. Scand J Med Sci Sports 1995:5:245-51.

3 Souza DR, Gross MT. Comparison of vastus medialis obliquus: vastus lateralis muscle integrated electromyographic ratios between healthy subjects and patients with patellofemoral pain. Phys Ther 1991;71:310-6.

4 Miller JP, Sedory D, Croce RV. Vastus medialis obliquus and vastus lateralis activity in patients with and without patellofemoral pain syndrome. J Sport Rehabil 1997;6:1-10

5 Callaghan MJ, McCarthy CJ, Oldham JA. Electromyographic fatigue characteristics of the quadriceps in patellofemoral pain syndrome. Man Ther $2001 ; 6: 27-33$

6 Tang SF, Chen CK, Hsu R, et al. Vastus medialis obliquus and vastus lateralis activity in open and closed kinetic chain exercises in patients with patellofemoral pain syndrome: an electromyographic study. Arch Phys Med Rehabil 2001;82:1441-5.

7 Voight ML, Wieder DL. Comparative reflex response times of vastus medialis obliquus and vastus lateralis in normal subjects and subjects with extensor mechanism dysfunction. An electromyographic study. Am J Sports Med 1991;19:131-7.

8 Karst GM, Willett GM. Onset timing of electromyographic activity in the vastus medialis oblique and vastus lateralis muscles in subjects with and without patellofemoral pain syndrome. Phys Ther 1995;75:813-23.

9 Powers CM, Landel R, Perry J. Timing and intensity of vastus muscle activity during functional activities in subjects with and without patellofemoral pain. Phys Ther 1996:76:946-55.

10 Witvrouw E, Sneyers C, Lysens R, et al. Reflex response times of vastus medialis oblique and vastus lateralis in normal subjects and in subjects with patellofemoral pain syndrome. J Orthop Sports Phys Ther 1996;24:160-5.
11 Wityrouw E, Lysens R, Bellemans J, et al Intrinsic risk factors for the development of anterior knee pain in an athletic population. A two-year prospective study. Am J Sports Med 2000;28:480-9.

12 Cesarelli M, Bifulco P, Bracale M. Study of the control strategy of the quadriceps muscles in anterior knee pain. IEEE Trans Rehabil Eng 2000;8:330-41.

13 Cowan SM, Bennell KL, Hodges PW, et al. Delayed onset of electromyographic activity of vastus medialis obliquus relative to vastus lateralis in subjects with patellofemoral pain syndrome. Arch Phys Med Rehabil 2001:82:183-9.

14 Hanten WP, Schulthies SS. Exercise effect on electromyographic activity of the vastus medialis oblique and vastus lateralis muscles. Phys Ther 1990:70:561-5

15 Grabiner MD, Koh RJ, Miller GF. Fatigue rates of the vastus medialis oblique and vastus lateralis during static and dynamic knee extension. J Orthop Res $1991 ; 9: 391-7$

16 Grabiner MD, Koh RJ, Von Haefen L. Effect of concomitant hip joint adduction and knee joint extension forces on quadriceps activation. Eur J Musculoskel Res 1992;1:155-60.

17 Karst GM, Jewett PD. Electromyographic analysis of exercises proposed for differential activation of medial and lateral quadriceps femoris muscle components. Phys Ther 1993;73:286-95.

18 Hodges PW, Richardson CA. The influence of isometric hip adduction on quadriceps femoris activity. Scand J Rehabil Med 1993;25:57-62.

19 Cerny K. Vastus medialis oblique/vastus lateralis muscle activity ratios for selected exercises in persons with and without patellofemoral pain syndrome. Phys Ther 1995;75:672-83.

20 Worrell T, Connelly S, Hilvert J. VMO:VL ratios and torque comparisons at four angles of knee flexion. J Sport Rehabil 1995;4:264-72.

21 Laprade J, Culham E, Brouwer B. Comparison of five isometric exercises in the recruitment of the vastus medialis oblique in persons with and without patellofemoral pain syndrome. J Orthop Sports Phys Ther 1998;27:197-204.

22 Monteiro-Pedro V, Vitti M, Berzin F, et al. The effect of free isotonic and maximal isometric contraction exercises of the hip adduction on vastus medialis oblique muscle: an electromyographic study. Electromyogr Clin Neurophysiol 1999;39:435-40.

23 Earl JE, Schmitz RJ, Arnold BL. Activation of the VMO and VL during dynamic mini-squat exercises with and without isometric hip adduction. J Electromyogr Kinesiol 2001;11:381-6.

24 Lam PL, $\mathrm{Ng}$ GY. Activation of the quadriceps muscle during semisquatting with different hip and knee positions in patients with anterior knee pain. Am J Phys Med Rehabil 2001;80:804-8.

25 Host J, Craig R, Lehman R. Patellofemoral dysfunction in tennis players: a dynamic problem. Clin Sports Med 1995; 14:177-203.

26 Sahrmann S. Muscle imbalances in the female athlete. In: Pearl A, ed The female athlete. Champaign, IL: Human Kinetics, 1993:209-218.

27 Soderberg GL, Knutson LM. A guide for the use and interpretation of kinesiologic electromyographic data. Phys Ther 2000;80:485-98.

28 Yamashita N. EMG activities in mono- and bi-articular thigh muscles in combined hip and knee extension. Eur J Appl Physiol Occup Physiol 1988; $58: 274-7$

29 Hase K, Stein RB. Turning strategies during human walking. J Neurophysiol 1999;81:2914-22.

30 Schmitz RJ, Riemann BL, Thompson T. Gluteus medius activity during isometric closed-chain hip rotation. J Sport Rehabil 2002;11:179-88. 